\title{
Milnacipran Inhibits Oxaliplatin-Induced Mechanical Allodynia through Spinal Action in Mice
}

\author{
Tsugunobu Andoh, Ryo Kitamura, and Yasushi Kuraishi* \\ Department of Applied Pharmacology, Graduate School of Medicine and Pharmaceutical Sciences; 2630 Sugitani, \\ Toyama 930-0194, Japan.
}

Received August 11, 2014; accepted October 3, 2014

\begin{abstract}
We investigated whether milnacipran, a serotonin-noradrenaline reuptake inhibitor, would have therapeutic effect on oxaliplatin-induced mechanical allodynia in mice. A single intraperitoneal injection of oxaliplatin $(3 \mathrm{mg} / \mathrm{kg})$ induced mechanical allodynia, which peaked on day 10 after injection and almost completely subsided by day 20 . Ten days post-oxaliplatin injection, the intraperitoneal administration of milnacipran $(3-30 \mathrm{mg} / \mathrm{kg})$ significantly and dose-dependently inhibited the established mechanical allodynia. Intrathecal injections of milnacipran (2.1-21 $\mu \mathrm{g} / \mathrm{site})$ also significantly and dose-dependently inhibited mechanical allodynia, but intracisternal and intracereboventricular injections at the same doses did not. The present results suggest that milnacipran is effective against oxaliplatin-induced mechanical allodynia and that the antiallodynic effect is mainly mediated by actions on the spinal cord.
\end{abstract}

Key words milnacipran; oxaliplatin; allodynia; intrathecal injection; spinal cord

Oxaliplatin, a third-generation platinum-based chemotherapeutic agent, is a key drug for the treatment of colorectal cancer. However, oxaliplatin frequently causes dose-limiting adverse effects such as acute neurotoxicity and chronic peripheral neuropathy. ${ }^{1)}$ The distressing symptoms of peripheral neuropathy include pain and dysesthesia. ${ }^{2)}$ Although the underlying mechanisms of oxaliplatin-induced peripheral neuropathy are not completely understood, it has been reported that oxalate, a metabolite of oxaliplatin, is involved in acute peripheral neurotoxicity, especially cold dysesthesia. ${ }^{3,4)}$ Several drugs, including calcium gluconate/magnesium sulfate, glutathione, carbamazepine, gabapentin, amifostine, acetyl-Lcarnitine, and $\alpha$-lipoic acid, have been used to manage oxaliplatin-induced peripheral neuropathy.,5) Although these drugs are effective against mild neuropathy, oxaliplatin-induced peripheral neuropathy is still difficult to treat. ${ }^{4,5)}$

We have recently found that goshajinkigan, a traditional herbal medicine, suppresses oxaliplatin-induced mechanical allodynia and that this effect is, in part, mediated by both descending noradrenergic and serotonergic systems. $\left.{ }^{6}\right)$ These findings imply that enhancement of descending monoaminergic systems can inhibit established mechanical allodynia. Although amitriptyline, which inhibits the reuptake of noradrenaline and serotonin, ${ }^{7}$ has long been used for the treatment of neuropathic symptoms, especially in postherpetic neuralgia and diabetic neuropathy, it does not prevent chemotherapy-induced peripheral neuropathy in humans. ${ }^{8)}$ In contrast, prophylactic administration of venlafaxine, a serotonin-noradrenaline reuptake inhibitor (SNRI) with high potency in inhibiting serotonin reuptake, ${ }^{7)}$ has been reported to reduce oxaliplatin-induced acute neurotoxicity including spontaneous dysesthesia and daily pain in humans. ${ }^{9)}$ Prophylactic administration of duloxetine, a balanced SNRI, ${ }^{7)}$ has also been reported to reduce the severity of pain after 12 weeks of treatment. ${ }^{10)}$ In this study, we examined whether milnacipran, another balanced SNRI ${ }^{7)}$ would inhibit established mechanical allodynia after oxaliplatin administration in mice. In rats with neuropathy induced by spinal nerve ligation, mechanical allodynia has been reported to be inhibited by intrathecal, but not systemic, administration of milnacipran. ${ }^{11)}$ Additionally, it has been reported that following spinal nerve ligation in mice, mechanical allodynia can be inhibited by systemic and intracerebroventricular injections of milnacipran. ${ }^{12)}$ Furthermore, it has been shown that systemic administration of milnacipran in mice does not affect allodynia induced by the chemotherapeutic agent paclitaxel. ${ }^{13)}$ Thus, the efficacy and mechanisms (including site of action) of the antiallodynic effect of milnacipran seem to differ depending on causative factors. Therefore, we examined the antiallodynic activity of milnacipran after systemic, intrathecal, intracisternal, and intracerebroventricular injections.

\section{MATERIALS AND METHODS}

Animals Male C57BL/6 mice (Japan SLC Inc., Hamamatsu) were used. Mice were 6 weeks of age and weighed 18 to $22 \mathrm{~g}$ at the start of the experiments. They were housed six per cage under controlled temperature $\left(21-23^{\circ} \mathrm{C}\right)$ and humidity $(45-65 \%)$. The room was lighted from 7:00 a.m. to 7:00 p.m. Food and water were available ad libitum. All procedures for animal experiments were approved by the committee for animal experiments at the University of Toyama (\#S-2010 PHA-10).

Materials Oxaliplatin (Sigma-Aldrich, St. Louis, MO, U.S.A.) was dissolved in 5\% glucose and administered intraperitoneally at a dose of $3 \mathrm{mg} / \mathrm{kg}$, which was selected from a published report ${ }^{14)}$ and according to its recommended clinical dose. Milnacipran hydrochloride (Sigma-Aldrich) was dissolved in physiological saline. Intraperitoneal injection of milnacipran was administered in a volume of $10 \mathrm{~mL} / \mathrm{kg}$. Intrathecal, intracisternal, and intracerebroventricular injections were administered to awake animals in a volume of $5 \mu \mathrm{L}^{15)}$ Doses of milnacipran refer to the salt.

Behavioral Test Mechanical allodynia in the hind paw was evaluated using a fine von Frey filament (North Coast Medical Inc., Morgan Hill, CA, U.S.A.). ${ }^{14)}$ After an acclima- 


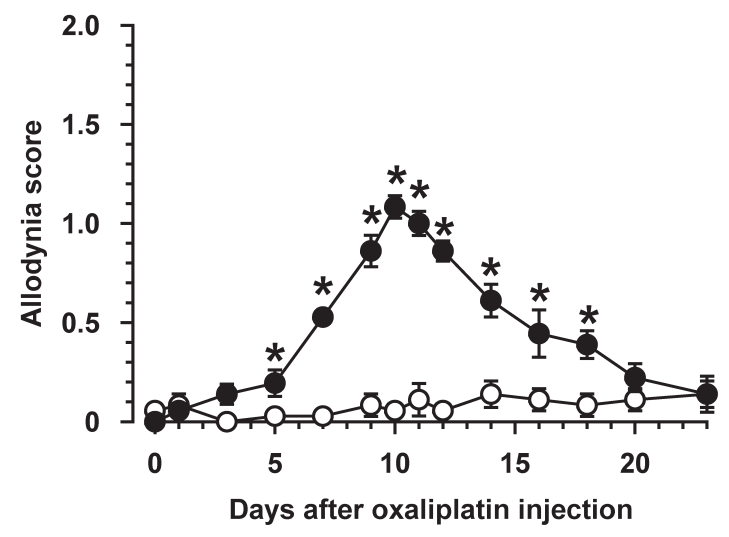

Fig. 1. Time Course of Mechanical Allodynia after a Single Injection of Oxaliplatin

Mice were given an intraperitoneal injection of oxaliplatin $(3 \mathrm{mg} / \mathrm{kg})$ or the vehicle on day 0 . Mechanical allodynia was tested by punctate stimulation using a von Frey filament of $0.69-\mathrm{mN}$ strength. Closed and open circles represent oxaliplatin and the vehicle, respectively. Data are presented as mean and standard error of the mean $(n=6)$. Main effect of oxaliplatin treatment, $F_{110}=166.4, p<0.001$; interaction between oxaliplatin treatment and time, $F_{13,130}=19.5, p<0.001$ (two-way repeated measures ANOVA). ${ }^{*} p<0.05$ compared to vehicle (post hoc Holm-Šidák multiple comparisons).

tion period of at least $30 \mathrm{~min}$, a von Frey filament with a bending force of $0.69 \mathrm{mN}$ was applied perpendicularly against the central part of the plantar hind paw and was held for $1-3 \mathrm{~s}$ with it slightly bent. Responses to the stimuli were scored as follows: 0 , no reaction; 1 , lifting of the hind paw; 2 , licking and flinching of the hind paw. A stimulus of the same intensity was applied three times alternately to each hind paw at intervals of several seconds, and the average response score served as the allodynia score (the maximum score was 2). ${ }^{14}$

Data Processing All data are presented as means and standard error of the mean. Statistical significance was analyzed using two-way repeated measures ANOVA and post hoc Holm-Šidák multiple comparisons; $p<0.05$ was considered statistically significant.

\section{RESULTS}

A single intraperitoneal injection of oxaliplatin $(3 \mathrm{mg} / \mathrm{kg})$ caused mechanical allodynia, which became apparent on day 5 after injection, peaked on day 10, and almost subsided by day 20 (Fig. 1). The following experiments were performed on day 10. Intraperitoneal administration of milnacipran (3-30 mg/kg) produced a significant and dose-dependent inhibition of oxaliplatin-induced mechanical allodynia (Fig. 2A). This inhibition peaked $30-60 \mathrm{~min}$ after administration and subsided by $120 \mathrm{~min}$. Intrathecal, but not intracisternal and intracerebroventricular, injection of milnacipran $(2.1-21 \mu \mathrm{g} /$ site) produced a significant and dose-dependent inhibition of oxaliplatin-induced mechanical allodynia (Figs. 2B-D). The effect of intrathecal milnacipran peaked $30 \mathrm{~min}$ after administration and subsided by $120 \mathrm{~min}$.

\section{DISCUSSION}

A single intraperitoneal injection of oxaliplatin $(3 \mathrm{mg} / \mathrm{kg})$ caused mechanical allodynia in mice, with the time course and intensity being similar to what has been previously reported. ${ }^{14)} \mathrm{We}$ verified that systemic injection of milnacipran (3-30 mg/kg) suppressed the mechanical allodynia that had
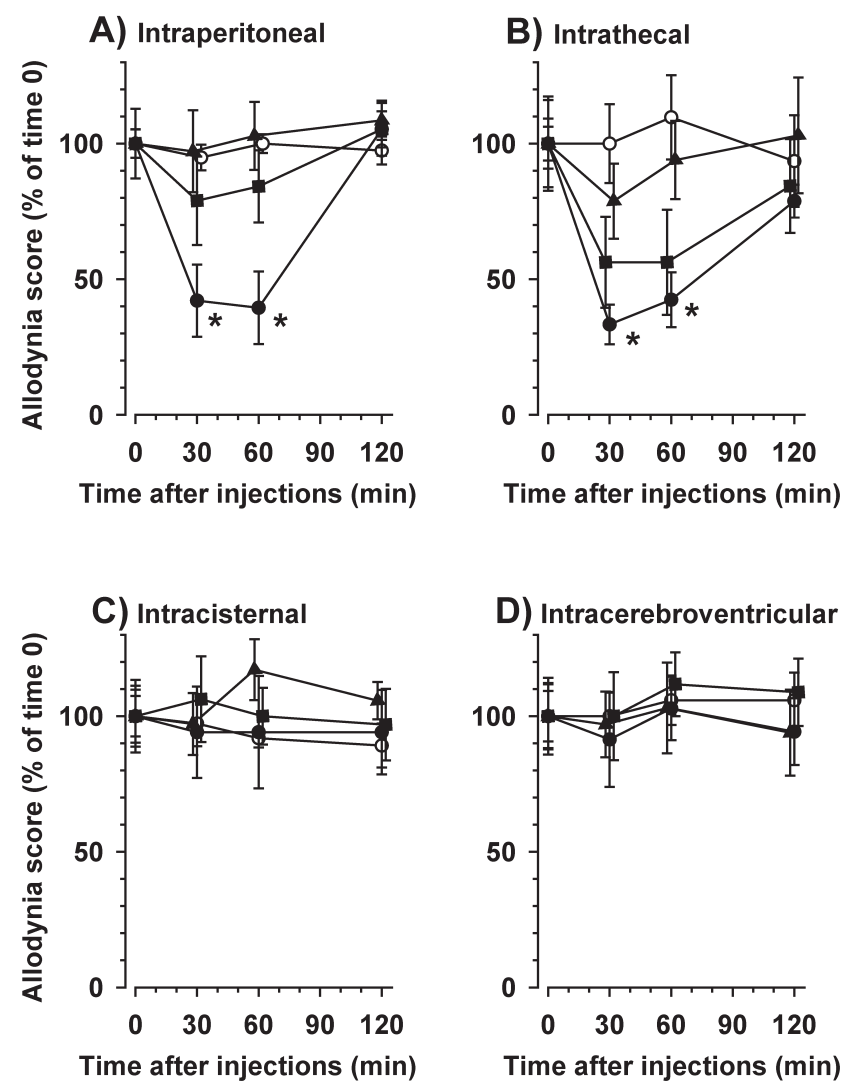

Fig. 2. Effects of Systemic and Local Injections of Milnacipran on Oxaliplatin-Induced Mechanical Allodynia

Oxaliplatin $(3 \mathrm{mg} / \mathrm{kg})$ was injected intraperitoneally (i.p.) into mice, and $10 \mathrm{~d}$ later milnacipran (closed symbols) or vehicle (open circles) was injected i.p. (A), intrathecally (B), intracisternally (C), or intracerebroventricularly (D). (A) The doses of milnacipran were 3 (closed triangles), 10 (closed squares), and $30 \mathrm{mg} / \mathrm{kg}$ (closed circles). (B-D) The doses of milnacipran were 2.1 (closed triangles), 7.0 (closed squares), and $21 \mu \mathrm{g} / \mathrm{site}$ (closed circles). Mechanical allodynia was examined before, 30,60 , and $120 \mathrm{~min}$ after milnacipran injection, as described in the legend for Fig. 1. Data are presented as mean and standard error of the mean $(n=6)$. (A) Interaction between milnacipran treatment and time, $F_{9,60}=4.71, p<0.001$; (B) Interaction between milnacipran treatment and time, $F_{9,60}=3.78, p<0.001$ (two-way repeated measures ANOVA). $* p<0.05$ compared to vehicle (post hoc Holm-Šidák multiple comparisons).

been induced by oxaliplatin. Regarding the site of action, intrathecal injections of milnacipran $(2.1-21 \mu \mathrm{g} / \mathrm{site})$ attenuated established allodynia. The degree of inhibition was similar between a systemic dose of $30 \mathrm{mg} / \mathrm{kg}$ and an intrathecal dose of $21 \mu \mathrm{g} / \mathrm{site}$. Given that the average body weight of the mice was $20 \mathrm{~g}$, a local dosage of $21 \mu \mathrm{g} / \mathrm{site}$ was equivalent to $1.05 \mathrm{mg} / \mathrm{kg}$, which was much smaller than the intraperitoneal equipotent dose $(30 \mathrm{mg} / \mathrm{kg})$. Therefore, the spinal cord may be an important site in the antiallodynic effect of intraperitoneal milnacipran.

Oxaliplatin-induced allodynia was significantly inhibited by milnacipran at an intrathecal dose of $21 \mu \mathrm{g} / \mathrm{site}$, and there was a decreased allodynic tendency at a dose of $7.0 \mu \mathrm{g} / \mathrm{site}$. In a previous report where mice that had undergone spinal nerve ligation, allodynia was inhibited by milnacipran at intrathecal doses of 7.0 and $21 \mu \mathrm{g} /$ site. $^{12)}$ Thus, antiallodynic potency of intrathecal milnacipran was similar between allodynia induced by oxaliplatin and spinal nerve ligation. Intrathecal milnacipran has also been shown to inhibit mechanical allodynia in rats that had undergone spinal nerve ligation or muscle incision. ${ }^{11,16)}$ The most probable mechanism underlying the antiallodynic effect of milnacipran's spinal action is the inhibition 
of noradrenaline and serotonin reuptake. Milnacipran is approximately twofold more potent in noradrenaline uptake inhibition than in serotonin uptake inhibition, although its binding affinity is higher to the serotonin transporter than the noradrenaline transporter. ${ }^{7,17)}$ When administered intravenously in naïve rats, milnacipran markedly increases extracellular noradrenalin concentration and to a lesser extent extracellular serotonin concentration. ${ }^{16)}$ Furthermore, the antiallodynic action of intrathecal milnacipran is markedly blocked by intrathecal injection of the $\alpha_{2}$-adrenoceptor antagonist idazoxan and partially by intrathecal injection of the $5-\mathrm{HT}_{2}$ receptor antagonist methysergide. ${ }^{16)}$ Taken together, these findings suggest that the descending noradrenergic and serotonergic pathways play key roles in the antiallodynic action of milnacipran.

Regarding intracerebroventricular administration in mice, milnacipran did not inhibit oxaliplatin-induced allodynia at doses of $21 \mu \mathrm{g} / \mathrm{site}$ or lower (present study). In contrast, intracerebroventricular milnacipran has been shown to inhibit spinal nerve ligation-induced allodynia at $0.7 \mu \mathrm{g} / \mathrm{site}_{\text {or }}$ higher. ${ }^{12)}$ Although reason(s) for these differences remains unclear, it is possible that the antiallodynic mechanisms of milnacipran are somewhat different depending on the etiology of mechanical allodynia. In the current study, we observed that oxaliplatininduced allodynia was not inhibited by intracisternal injections of milnacipran. The locus coeruleus in the pons is an important nucleus of origin of the descending noradrenergic system $^{18,19)}$ and is easily affected by intracisterally injected agents. ${ }^{20)}$ Spontaneous activity of neurons in the locus coeruleus is inhibited by systemic injections of venlafaxine, ${ }^{21)}$ duloxetine, ${ }^{22}$ and reboxetine (noradrenaline reuptake inhibitor). ${ }^{23)}$ Furthermore, the activity of locus coeruleus neurons is also inhibited by local administration of citalopram (serotonin reuptake inhibitor) in the vicinity of the locus coeruleus. ${ }^{24)} \mathrm{A}$ decrease in neural activity in the locus coeruleus may cause the decrease of the activity of the descending noradrenergic system, leading to a decrease in noradrenaline release from its terminals in the dorsal horn. Thus, it may not produce antiallodynia but instead may counteract antiallodynic activity of the antidepressants.

Established mechanical allodynia $10 \mathrm{~d}$ after oxaliplatin injection was inhibited by milnacipran at an intraperitoneal dose of $30 \mathrm{mg} / \mathrm{kg}$ (present study), while acute cold hypersensitivity $2 \mathrm{~h}$ after oxaliplatin injection was shown not to be inhibited by milnacipran at the same intraperitoneal dose. ${ }^{25}$ Although reasons for these differences are unclear, it is possible that efficacy of milnacipran is different between oxaliplatin-induced cold hypersensitivity and chronic neuropathy. In humans treated with oxaliplatin, venlafaxine inhibits worst daily pain and pins-and-needles, but not cold-triggered pain. ${ }^{9)}$ In rats, daily administration of amitriptyline inhibits oxaliplatininduced mechanical allodynia but not cold hypersensitivity. ${ }^{26}$ These findings raised the possibility that oxaliplatin-induced cold hypersensitivity is refractory to antidepressants including milnacipran.

In summary, the present results suggest that milnacipran is effective against established mechanical allodynia after oxaliplatin treatment. The inhibitory action of milnacipran on oxaliplatin-induced allodynia may be mainly mediated by actions on the spinal cord, but not the brain regions.

Acknowledgments This research was supported by a
Grant-in-Aid for the Cooperative Research Project from Joint Usage/Research Center (Joint Usage/Research Center for Science-Based Natural Medicine), Institute of Natural Medicine, University of Toyama in 2011 and Takeda Science Foundation 2009.

Conflict of Interest The authors declare no conflict of interest.

\section{REFERENCES}

1) Park SB, Goldstein D, Krishnan AV, Lin CS, Friedlander ML, Cassidy J, Koltzenburg M, Kiernan MC. Chemotherapy-induced peripheral neurotoxicity: A critical analysis. CA Cancer J. Clin., 63, 419-437 (2013).

2) Quasthoff S, Hartung HP. Chemotherapy-induced peripheral neuropathy. J. Neurol., 249, 9-17 (2002).

3) Sakurai M, Egashira N, Kawashiri T, Yano T, Ikesue H, Oishi R. Oxaliplatin-induced neuropathy in the rat: involvement of oxalate in cold hyperalgesia but not mechanical allodynia. Pain, 147, 165-174 (2009).

4) Pasetto LM, D'Andrea MR, Rossi E, Monfardini S. Oxaliplatinrelated neurotoxicity: how and why? Crit. Rev. Oncol. Hematol., 59, 159-168 (2006).

5) Saif MW, Reardon J. Management of oxaliplatin-induced peripheral neuropathy. Ther. Clin. Risk Manag., 1, 249-258 (2005).

6) Kitamura R, Andoh T, Fushimi H, Komatsu K, Shibahara N, Kuraishi Y. Involvement of descending monoaminergic systems in antiallodynic effect of goshajinkigan in oxaliplatin-treated mice. $J$. Trad. Med., 30, 183-189 (2013).

7) Vaishnavi SN, Nemeroff CB, Plott SJ, Rao SG, Kranzler J, Owens MJ. Milnacipran: a comparative analysis of human monoamine uptake and transporter binding affinity. Biol. Psychiatry, 55, 320-322 (2004).

8) Kautio AL, Haanpaa M, Leminen A, Kalso E, Kautiainen H, Saarto T. Amitriptyline in the prevention of chemotherapy-induced neuropathic symptoms. Anticancer Res., 29, 2601-2606 (2009).

9) Durand JP, Deplanque G, Montheil V, Gornet JM, Scotte F, Mir O, Cessot A, Coriat R, Raymond E, Mitry E, Herait P, Yataghene Y, Goldwasser F. Efficacy of venlafaxine for the prevention and relief of oxaliplatin-induced acute neurotoxicity: results of EFFOX, a randomized, double-blind, placebo-controlled phase III trial. Ann. Oncol., 23, 200-205 (2012).

10) Yang YH, Lin JK, Chen WS, Lin TC, Yang SH, Jiang JK, Chang SC, Lan YT, Lin CC, Yen CC, Tzeng CH, Wang WS, Chiang HL, Teng CJ, Teng HW. Duloxetine improves oxaliplatin-induced neuropathy in patients with colorectal cancer: an open-label pilot study. Support. Care Cancer, 20, 1491-1497 (2012).

11) King T, Rao S, Vanderah T, Chen Q, Vardanyan A, Porreca F. Differential blockade of nerve injury-induced shift in weight bearing and thermal and tactile hypersensitivity by milnacipran. J. Pain, 7, 513-520 (2006).

12) Suzuki T, Ueta $K$, Tamagaki S, Mashimo T. Antiallodynic and antihyperalgesic effect of milnacipran in mice with spinal nerve ligation. Anesth. Analg., 106, 1309-1315 (2008).

13) Katsuyama S, Sato K, Yagi T, Kishikawa Y, Nakamura H. Effects of repeated milnacipran and fluvoxamine treatment on mechanical allodynia in a mouse paclitaxel-induced neuropathic pain model. Biomed. Res., 34, 105-111 (2013).

14) Gauchan P, Andoh T, Kato A, Sasaki A, Kuraishi Y. Effects of the prostaglandin E1 analog limaprost on mechanical allodynia caused by chemotherapeutic agents in mice. J. Pharmacol. Sci., 109, 469472 (2009).

15) Takasaki I, Andoh T, Nojima H, Shiraki K, Kuraishi Y. Gabapentin antinociception in mice with acute herpetic pain induced by herpes 
simplex virus infection. J. Pharmacol. Exp. Ther, 296, 270-275 (2001).

16) Obata H, Kimura M, Nakajima K, Tobe M, Nishikawa K, Saito S. Monoamine-dependent, opioid-independent antihypersensitivity effects of intrathecally administered milnacipran, a serotonin noradrenaline reuptake inhibitor, in a postoperative pain model in rats. J. Pharmacol. Exp. Ther., 334, 1059-1065 (2010).

17) Moret C, Charveron M, Finberg JP, Couzinier JP, Briley M. Biochemical profile of midalcipran (F 2207), 1-phenyl-1-diethyl-aminocarbonyl-2-aminomethyl-cyclopropane $(Z)$ hydrochloride, a potential fourth generation antidepressant drug. Neuropharmacology, 24, 1211-1219 (1985)

18) Millan MJ. Descending control of pain. Prog. Neurobiol., 66, $355-$ 474 (2002)

19) Yoshimura M, Furue H. Mechanisms for the anti-nociceptive actions of the descending noradrenergic and serotonergic systems in the spinal cord. J. Pharmacol. Sci., 101, 107-117 (2006).

20) Tibiriça E, Feldman J, Mermet C, Gonon F, Bousquet P. An imidazoline-specific mechanism for the hypotensive effect of clonidine: a study with yohimbine and idazoxan. J. Pharmacol. Exp. Ther., 256, 606-613 (1991).

21) Berrocoso E, Mico JA. In vivo effect of venlafaxine on locus coeruleus neurons: role of opioid, $\alpha_{2}$-adrenergic, and 5-hydroxytryptamine receptors. J. Pharmacol. Exp. Ther., 322, 101-107 (2007).

22) Alba-Delgado C, Mico JA, Sanchez-Blazquez P, Berrocoso E. Analgesic antidepressants promote the responsiveness of locus coeruleus neurons to noxious stimulation: implications for neuropathic pain. Pain, 153, 1438-1449 (2012).

23) Bruzos-Cidón C, Miguelez C, Rodriguez JJ, Gutierrez-Lanza R, Ugedo L, Torrecilla M. Altered neuronal activity and differential sensitivity to acute antidepressants of locus coeruleus and dorsal raphe nucleus in Wistar Kyoto rats: A comparative study with Sprague Dawley and Wistar rats. Eur. Neuropsychopharmacol., 24, 1112-1122 (2014)

24) Mateo Y, Ruiz-Ortega JA, Pineda J, Ugedo L, Meana JJ. Inhibition of 5-hydroxytryptamine reuptake by the antidepressant citalopram in the locus coeruleus modulates the rat brain noradrenergic transmission in vivo. Neuropharmacology, 39, 2036-2043 (2000).

25) Zhao M, Nakamura S, Miyake T, So K, Shirakawa H, Tokuyama S, Narita M, Nakagawa T, Kaneko S. Pharmacological characterization of standard analgesics on oxaliplatin-induced acute cold hypersensitivity in mice. J. Pharmacol. Sci., 124, 514-517 (2014).

26) Sada H, Egashira N, Ushio S, Kawashiri T, Shirahama M, Oishi R. Repeated administration of amitriptyline reduces oxaliplatininduced mechanical allodynia in rats. J. Pharmacol. Sci., 118 547-551 (2012). 